\title{
Image Inpaint Using Patch Sparsity
}

\author{
Shital D. Suryawanshi*, P. V. Baviskar \\ Department of Electronics, SSVPS COE, Dhule, (MS), India \\ *Corresponding author, e-mail: ssuryawanshi9@gmail.com, baviskarpv@rediffmail.com
}

\begin{abstract}
The process of removing the specific object or area or repairing the damaged area in an image is known as image inpainting. This algorithm [5] is proposed for removing objects from digital image. The challenge is to fill in the hole that is left behind in a visually plausible way. We first note that patch sparsity based synthesis contains the essential process required to replicate both texture and structure [8]; the success of structure propagation however is highly dependent on the order in which the filling proceeds. We propose a best algorithm in which the confidence in the synthesized pixel values is propagated in a manner similar to the propagation of information in inpainting.The actual color values are computed using patch sparsity based synthesis. In this paper the simultaneous propagation of texture and structure information [2] is achieved by a single, efficient algorithm. For best results selected image should have sufficient background information.
\end{abstract}

Keywords: Inpainting Algorithm, Region Filling, Confidence, Patch Propagation, Patch Sparsity

Copyright (C) 2016 APTIKOM - All rights reserved.

\section{Introduction}

Image inpainting is an interesting topic in computer graphics [16] and an active area in research in image processing. Image inpainting is also known as image interpolation or completion. Object removal from image [14] is an image manipulation technique that has a long history and the purpose of removing objects varies from removing undesired object to improve the quality of the image [19], to airbrushing out political enemies from portraits of important events. Modern photographical manipulations, such as red eye removal [19] from pictures, also utilized this technique. It is used in video inpainting also to correct videos.

The purpose of inpainting is to filling in the missing part of an image [1]. Diffusion based inpainting preserves the structure (i.e. lines and object contours) by propagating the isophote (a line of equal luminance) in the unknown region [1]. By construction this approach achieves excellent results when the missing region is small. This problem occurs when block of data have been lost in transmission [1], or intentionally pruned for compression, or when an unwanted object has to be removed. In this context the missing part contains both image structure and texture [1]. State of art methods are patch sparsity based inpainting and were inspired by texture synthesis algorithms.Inpainting using patch sparsity fills the holes in an image by searching similar information on the known region in terms of patch and simply copies it to the unknown region as is done in texture synthesis. The previous idea is based on the fact that natural images contain redundant or very similar information. Greedy inpainting using exemplars also known as patches [10] consist of two major steps select the patch to be filled and propagate the texture and structure. The former step selects patches with mostly linear structures by giving them higher priority. The later is related to the selection of the most similar patches from which the information is copied. These two steps are iterated until the holes in the image are fully restored.Image inpainting applications are in repairing photographs to remove unwanted objects. This may be used in producing special effect in images and videos [15].

\section{Research Method}

One of the earliest modes of image inpainting was in usage of general texture synthesis algorithms [12] to complete the missing regions Efros \& Leung (1999) utilized these methods to fill the holes by sampling and copying pixels from neighboring areas. For examining literature of Efros \& Leung (1999), Markov Random Field (MRF) is used to model the local distribution of a pixel and new texture is 
synthesized by querying existing texture and finding all similar neighborhoods [12]. The second group is named as PDE inpainting. It was first introduced by Bertalmio etal (2000). Their method propagates image Laplacians from the known areas of the image inwards in the isophote direction. The drawback of the inpainting technique proposed by Bertalmio etal (2000) is that the reconstruction of large textured regions is impossible and the diffusion process produces blurring. Criminisi etal (2004) proposed a priority order to emphasize in Propagation of linear structures.

\subsection{Inpainting Algorithm Classification According To the Geometric Approach \\ 2.1.1. Structural Inpainting}

Structure synthesis means to fill in the missing information in such a way that isophote lines arriving at the region's boundaries are completed inside [6]. These methods allow for simultaneous filling in of multiple regions containing completely different structure and surrounding backgrounds. If structure synthesis is done using PDE based methods it introduce blur in the image.

\subsubsection{Textural Inpainting}

Like everything else the structural inpainting methods have both advantages and disadvantages. The main problem is that all the structural inpainting methods are not able to restore texture [4]. Texture has a repetitive pattern which means that a missing portion cannot be restored by continuing the level lines into the gap. The problem of texture inpainting is highly connected with the problem of texture synthesis [9]. A very simple and highly effective algorithm was presented by Efros and Leung. In this algorithm the image is modeled as a Markov Random Field and texture is synthesized in a pixel by pixel way by picking existing pixels with similar neighborhoods in a randomized fashion. This algorithm performs very well but it is very slow since the filling in is being done pixel by pixel.

\subsubsection{Combined Structural and Textural Inpainting}

Combined structural and textural inpainting approaches simultaneously try to perform texture and structure filling in regions of missing image information [4]. Most parts of an image consist of texture and structure. The boundaries between image regions accumulate structural information which is a complex phenomenon. This is the result when blending different textures together. That is why the state of the art inpainting method attempts to combine structural and textural inpainting.A more traditional method is to use differential equations (such as the Laplace's equation) with Dirichlet boundary conditions for continuity. This works well if missing information lies within the homogeneous portion of an object area.

\subsection{Inpainting Algorithm Is Classified Into Two Basic Categories According to Operation 2.2.1. Inpainting Algorithm Based On PDE (Partial Differential Equations)}

The algorithm based on PDE's (Partial Differential Equations) [12] which propagates the information along the isophotes (contour line) at the boundary on pixel level by diffusion.PDE based algorithm is used to repair small size region of digital image.PDE's algorithm for small regions, a mathematical method has been introduced by M. Bertalmio etal. The method is propagating the geometric structure of the image. The PDE based inpainting algorithms have drawback that it introduces some blur in the image after filling algorithm. It cannot fill the large missing region [16] and it cannot renovate the texture pattern.

\subsubsection{Inpainting Algorithm Based on Using Patch Sparsity}

This paper presents patch sparsity based algorithm for removing large objects from digital photographs and replacing them with visually plausible backgrounds [17]. The algorithm effectively hallucinates new color values for the target region in a way that looks "reasonable" to the human eye.

\section{Proposed Work}

This algorithm is proposed for removing objects [5] from digital image. The challenge is [2] to fill in the hole that is left behind in a visually plausible way. It replicates both texture and structure; the success of structure propagation however is highly dependent on the order in which the filling proceeds [2]. We propose a best algorithm in which the confidence in the synthesized pixel values is propagated in a manner similar to the propagation of information in inpainting.

APTIKOM J. CSIT Vol. 1, No. 3, 2016 : 85 - 91 


\subsection{Block Diagram Explanation Of Image Inpaint Using Patch Sparsity}

The input image file is taken in that the object we have to remove is selected (target). Target can be selected by the user as per his requirement using the cropping tool in Matlab. After selecting the target region apply the proposed method for removing that respective object. The process is done by using best matching patch [11] that from the source region is inpainted in target region so as to fill the holes. Here the most similar [15] patch is searched from the source region $\Phi$ to compose the given patch, $\Psi$ (of size $\mathrm{N}$ $\times \mathrm{N}$ pixels) that centered on the given pixel P.Filling is done in unfilled region in target to replace the pixels then values are updated to get the new values of pixel and we get the new boundaries for image the process continues till whole target region gets filled. After getting the entire region filled we get the final inpainted output which is a new image which we desire. Figure 1 shows block diagram for proposed system.

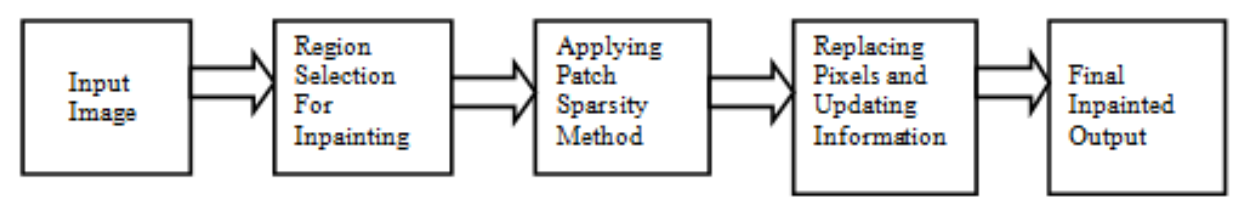

Figure 1. Block Diagram for Proposed System

\subsection{Image Inpaint Using Patch Sparsity}

The core of our algorithm [2] is an isophote driven image sampling process. It is well understood that this approach perform well for 2D textures. However, we note in addition that patch based texture synthesis is sufficient for propagating extended linear image structures as well i.e. separate synthesis mechanism is not required for handling isophotes. The region to be filled [10] i.e. the target region is indicated by $\Omega$, and its contour is denoted by $\partial \Omega$.

The contour evolves inward as the algorithm [2] progresses, and so we also refer to it as the "fill front". The source region $\Phi$ which remains fixed throughout the algorithm provides samples used in the filling process. We now focus on a single iteration of the algorithm to show how structure and texture are adequately handled by patch synthesis. Suppose that the square template centered at the point (Figure 2(b)) is to be filled. The best match sample from the source region comes from the patch which is most similar to those parts that are already filled in. In the example in Figure 2. (b), we see that if lies on the continuation of an image edge, the most likely best matches will lie along the same (or a similarly colored) edge (Figure 3(a)).

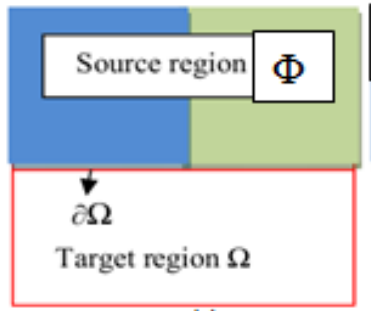

(a)

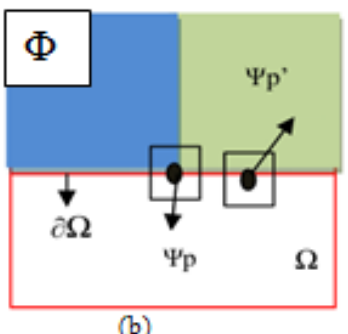

(b)

Figure 2. (a) image with target $\Omega, \Phi$ is the source region and $\partial \Omega$ is the fill front (b) Two patches $\Psi p$ and $\Psi \mathrm{p}$ '

Now the Figure 3(a) Shows [13] that for the selected patch $\Psi$ p, sparse linear combination of candidate patches $\left\{\Psi \mathrm{p},, \Psi \mathrm{p},{ }^{\prime} \ldots \Psi \mathrm{p}_{\mathrm{N}}\right\}$ is used to fill the missing pixels in patch $\Psi \mathrm{p},(\mathrm{b})$ Shows the best matching patch in the candidates set has been copied into the position occupied by $\Psi \mathrm{p}$, thus achieving partial filling of $\Omega$. All that is required [2] to propagate the isophote inwards is a simple transfer of the pattern from the best match source patch (Figure 3(b)). Notice that isophote orientation is automatically preserved. In the figure despite the fact that the original edge is not orthogonal to the target contour the 
propagated structure has maintained the same orientation as in the source region. In this work we focus on a patch based filling approach because as noted this improves execution speed [1]. Furthermore we note that patch based filling improves the accuracy of the propagated structures.

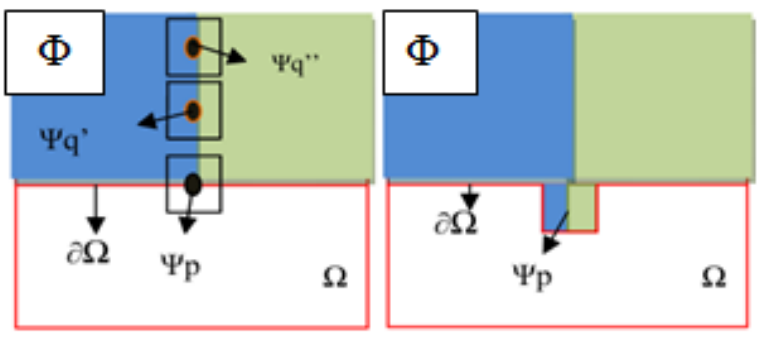

(a)

Figure 3. Patch Inpainting

First given an input image the user selects a target region $\Omega$ to be removed [3] and filled. The source region $\Phi$ may be defined as the entire image minus the target region $(\Phi=\mathrm{I}-\Omega)$ as a dilated band around the target region, or it may be manually specified by the user. Next as with all exemplar based texture synthesis the size of the template window must be specified. We provide three window size here of $5 \times 5,7 \times 7$ and $9 \times 9$ pixels. Once these parameters [1] are determined, the region filling proceeds automatically. In our algorithm each pixel maintains a color value (or "empty" if the pixel is unfilled) and a confidence value which reflects our confidence in the pixel value and which is frozen once a pixel has been filled. During the course of the algorithm patches along the fill front are also given a temporary priority value which determines the order in which they are filled. Then our algorithm iterates the following three steps until all pixels have been filled.

\subsubsection{Computing Patch Priorities}

Our algorithm performs the synthesis task through a best first filling strategy that depends entirely on the priority values that are assigned to each patch on the fill front. The priority computation is based toward those patches which are on the continuation of strong edges and are surrounded by high confidence pixels. Given a patch $\Psi$ p centered at the point $\mathrm{P}$ for some $\mathrm{P} \in \partial \Omega$, we define its priority $\mathrm{P}(\mathrm{p})$ as the product of two terms

$$
P(p)=C(p) D(p)
$$

We call C (p) the confidence term and D (p) the data term and they are defined as follows

$$
C(\mathbf{p})=\frac{\sum_{\mathbf{q} \in \Psi_{\mathbf{p}}} \bigcap(\mathcal{I}-\Omega)}{\left|\Psi_{\mathbf{p}}\right|} \quad D(\mathbf{q})=\frac{\left|\nabla I_{\mathbf{p}}^{\perp} \cdot \mathbf{n}_{\mathbf{p}}\right|}{\alpha}
$$

Where $|\Psi \mathrm{p}|$ is the area of $\Psi \mathrm{p}$, is $\alpha$ a normalization factor (e.g., $\alpha=255$ for a typical grey level image), is $n_{p}$ a unit vector orthogonal to the front $\partial \Omega$ in the point $P$. The priority $P(p)$ is computed for every border patch with distinct patches for each pixel on the boundary of the target region. The confidence term may be thought [7] of as a measure of the amount of reliable information surrounding the pixel. The intention is to fill first those patches which have more of their pixels already filled with additional preference given to pixels that were filled early on (or that were never part of the target region) . For example patches that include corners and thin tendrils of the target region will tend to be filled first as they are surrounded by more pixels from the original image. These patches provide more reliable information against which to match the term approximately enforces the desirable concentric fill order [3]. As filling proceeds pixels in the outer layers of the target region will tend to be characterized by greater confidence values and therefore be filled earlier; pixels in the center of the target region will have lesser confidence values. The data term $\mathrm{D}(\mathrm{p})$ is a function of the strength of isophotes hitting the front $\partial \Omega$ at each iteration. This term boosts the priority of a patch that an isophote "flows" into. This factor is

APTIKOM J. CSIT Vol. 1, No. 3, $2016: 85$ - 91 
of fundamental importance in our algorithm because it encourages linear structures to be synthesized first and therefore propagated securely into the target region. Broken lines tend to connect thus realizing the "connectivity principle" of vision psychology.

\subsubsection{Propagating Texture and Structure Information}

Once all priorities on the fill front have been computed [5] the patch $\Psi p$ with highest priority is found. We then fill it with data extracted from the source region $\Phi$.

\subsubsection{Updating Confidence Values}

After the patch $\Psi \mathrm{p}$ has been filled with new pixel values, the confidence $\mathrm{C}(\mathrm{p})$ is updated in the area delimited by $\Psi \mathrm{p}$ as follows [11]

$$
C(\mathbf{p})=C(\hat{\mathbf{p}}) \quad \forall \mathbf{p} \in \Psi_{\hat{\mathbf{p}}} \bigcap \Omega
$$

This simple update rule allows us to measure the relative confidence of patches on the fill front without image specific parameters. As filling proceeds confidence values decay indicating that we are less sure of the color values of pixels near the center of the target region.

\section{Result}

In Figure 4 the original image contains five dogs and in output image one is removed and filled with background pixels. In Figure 5 the original image contains text 'DREAM BIG' which is removed in output image. Figure 6 contains red colored tree and Figure 7 contains green tree both are removed in respective output images. Four images are inpainted by using the patch sparsity based inpainting algorithm and the different parameters like Time, PSNR, MSE and correlation are find out. For each image three different patch sizes are selected and it is observed that as the patch size increases time required to execute the program is reduced it improves the performance. The PSNR values are in the range of 40 to $50 \mathrm{db}$ which shows that image is having less noise and its quality is good.MSE is in range 1 to $5 \mathrm{db}$ which is acceptable both are reciprocal to each other.
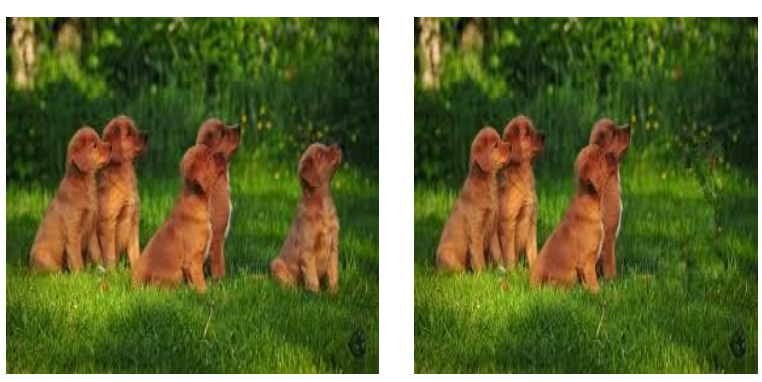

Figure 4. Image 1
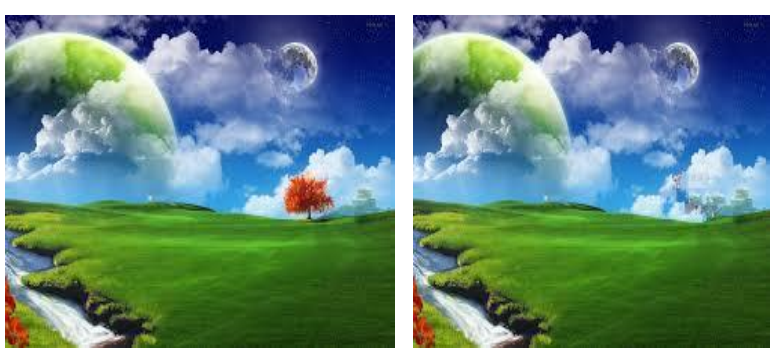

Figure 6. Image 3
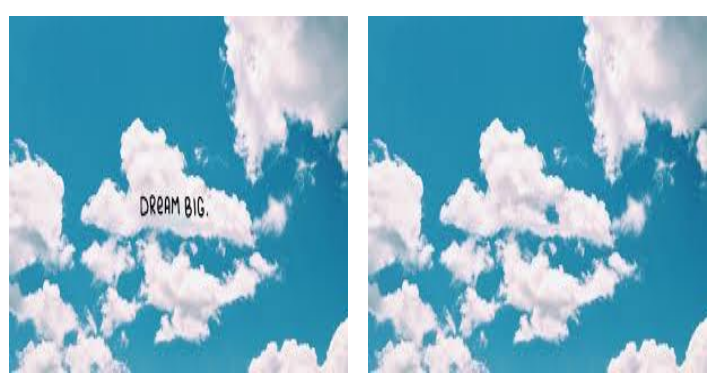

Figure 5. Image 2
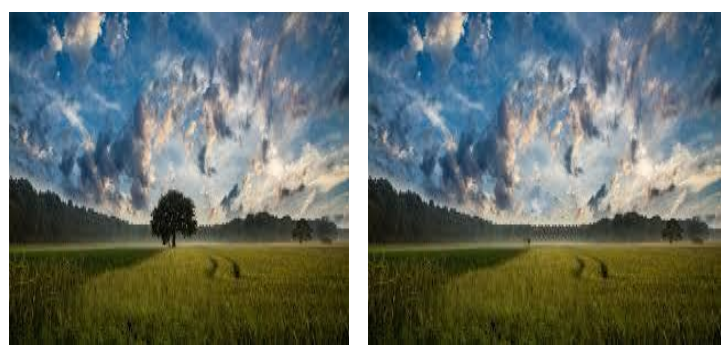

Figure 7. Image 4 
Table 1. Practical Values of Different Parameters of Image Inpaint Using Patch Sparsity Algorithm

\begin{tabular}{lccccc}
\hline IMAGE & PATCH & $\begin{array}{c}\text { TIME } \\
(\mathrm{sec})\end{array}$ & $\begin{array}{c}\text { PSNR } \\
(\mathrm{db})\end{array}$ & MSE & CORRELATION \\
\hline \multirow{3}{*}{ IMAGE 1 } & $5 \times 5$ & 11.56 & 42.0894 & 4.0192 & 0.9326 \\
& $9 \times 7$ & 8.61 & 43.6361 & 2.8149 & 0.9197 \\
& $5 \times 9$ & 7.14 & 42.7494 & 3.4525 & 0.9261 \\
IMAGE 2 & $7 \times 7$ & 2.96 & 45.6038 & 1.7894 & 0.9599 \\
& $9 \times 9$ & 2.44 & 45.7672 & 1.7233 & 0.9609 \\
& $5 \times 5$ & 2.78 & 45.2214 & 1.9541 & 0.9557 \\
IMAGE 3 & $7 \times 7$ & 2.11 & 43.4377 & 2.9465 & 0.9799 \\
& $9 \times 9$ & 2.10 & 44.1383 & 2.5075 & 0.9814 \\
& $5 \times 5$ & 4.38 & 41.5516 & 4.5491 & 0.9696 \\
IMAGE 4 & $7 \times 7$ & 3.27 & 41.5976 & 4.5011 & 0.9629 \\
& $9 \times 9$ & 2.93 & 41.6335 & 4.4641 & 0.9642 \\
\hline
\end{tabular}

\section{Conclusion}

The PDE based inpainting algorithm cannot fill the missing region properly and it cannot renovate the texture pattern, the resulting image is having blur which is easily visible to the eye. The PSNR value is high and MSE is low for patch sparsity based method as compared to PDE based method which shows that patch sparsity based method is good. The analysis proves that the image inpaint using patch sparsity will create better results for inpainting the missing region also that this algorithm can inpaint textured image efficiently. This paper has presented a novel algorithm for removing large objects from digital photographs. The result is an image in which the selected object has been replaced by a visually plausible background that mimics the appearance of the source region [1].

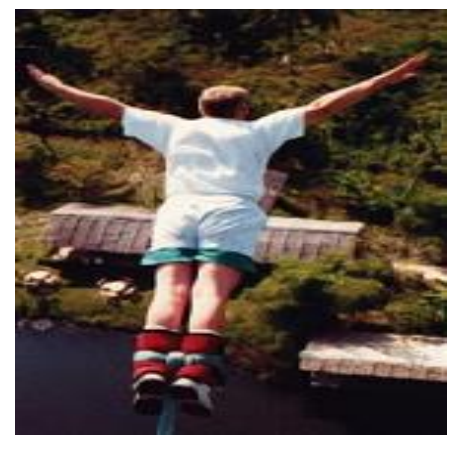

(a)

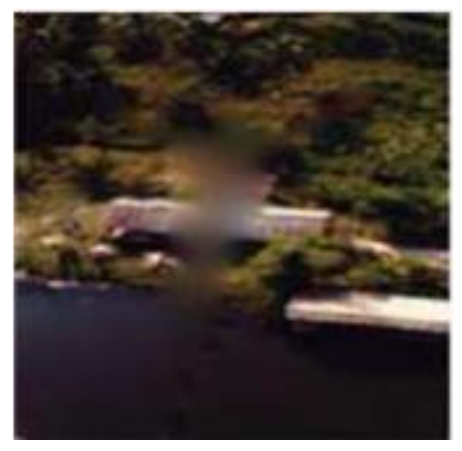

(b)

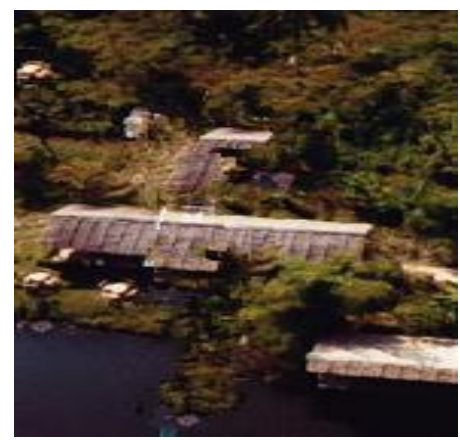

(c)

Figure 8. Comparison of results of PDE based method and Patch Sparsity based method (a) Original image (b) PDE result(c) Proposed method

Table 2. PDE Based Method

\begin{tabular}{ccc}
\hline PARAMETERS & PDE BASED & PATCH SPARSITY \\
& METHOD & BASED METHOD \\
\hline PSNR & 29.87 & 37.01 \\
MSE & 66.91 & 12.91 \\
CORRELATION & 0.23 & 0.65 \\
\hline
\end{tabular}

\section{References}

[1] R. Martinez, Noriega, A. Roumy, G. Blanchard "Exemplar Based Image Inpainting: Fast Priority And Coherent Nearest Neighbor Search" 2012 IEEE International Workshop On Machine Learning For Signal Processing, Sept. 23-26, 2012, Santander, Spain

[2] Antonio Criminisi, Patrick Perez, and Kentaro Toyama "Region Filling and Object Removal by Exemplar Based Image Inpainting" in IEEE Transactions on Image processing, vol. 13, no. 9, September 2004

[3] M. Bertalmio, L. Vese, G. Sapiro, and S. Osher, “ Simultaneous structure and texture image inpainting," IEEE Trans. Image Process., vol. 12, pp. 882-889, 2003.

APTIKOM J. CSIT Vol. 1, No. 3, 2016: 85 -91 
[4] Zongben Xu and Jian Sun "Image Inpainting by Patch Propagation Using Patch Sparsity" in IEEE Transactions on Image Processing, vol. 19, no. 5, MAY 2010.

[5] Jino Lee, Dong Kyu Lee, and Rae-Hong Park, "Robust Exemplar-Based Inpainting Algorithm Using Region Segmentation" in IEEE Transactions on Consumer Electronics, Vol. 58, No. 2, May 2012.

[6] Bagadiya Vishal, Prof.B.A.Dixit," Comparative Study and Analysis of Image Inpainting Techniques" IOSR Journal of Computer Engineering (IOSR-JCE) e-ISSN: 2278-0661, p- ISSN: 2278-8727Volume 15, Issue 4 (Nov. - Dec. 2013), PP 43-52.

[7] Shivali Tyagi, Sachin Singh "Image Inpainting By Optimized Exemplar Region Filling Algorithm" International Journal of Soft Computing and Engineering (IJSCE) ISSN: 2231-2307, Volume-2, Issue-6, January 2013.

[8] A. Efros and T. Leung, "Texture synthesis by non parametric sampling," in Proc. Int. Conf. Comp. Vision, 1999, pp. 1033-1038.

[9] R. Bornard, E. Lecan, L. Laborelli, and J. H. Chenot, "Missing data correction in still images and image sequences," in ACM Multimedia, France, Dec. 2002.

[10] Subban, Ravi, Muthukumar Subramanian, Pasupathi Perumalsamy, R. Seejamol, S. Gayathri Devi, and S.Selvakumar. "Tampered Image Reconstruction with Global SceneAdaptive Inpainting", Advances in Intelligent Systems and Computing, 2014.

[11] Devidas Lokhande, R.G.Zope, Vrushali Bendre "Image Inpainting" IJCSN International Journal of Computer Science and Network, Volume 3, Issue 1, February 2014 ISSN (Online): 2277-5420.

[12] Sudhir S.Kanade, Sandip S. Gujare "Comparative Study Of Different Digital Inpainting Algorithms" Volume 5 , Issue 12, December (2014), pp. 258-265communication Engineering \& Technology (IJECET).

[13] B.Vidhya, S. Valarmathy "Novel Video Inpainting Using Patch Sparsity "IEEE International Conference on Recent Trends in Information Technology, ICRTIT 2011978-1-4577-0590-8/11/\$26.00 @2011 IEEEMIT, Anna University, Chennai. June 3-5, 2011.

[14] Mrs.B.A.Ahire, Prof.Neeta A. Deshpande"Video inpainting of objects using modified Patch based technique", IJETCAS 13-636; () 2013.

[15] A.D.Boloorani, S. Erasmi, and M. Kappas, "Multi-Source Remotely Sensed Data Combination: Projection Transformation Gap-Fill Procedure," Sensor, pp. 84429-4440, July2008.

[16] P. Harrison, “A non-hierarchical procedure for resynthesis of complex texture.” In Pro G. Int. Conf Central Europe Camp. Graphics, Visual and Camp. Vision, Plzen, Czezh Republic, Feb 2001.

[17] Geeta K. Sarpate, Shanti K. Guru "Digital Image Inpainting Using Patch Priority Based Method "International Journal of Advance Foundation and Research in Computer (IJAFRC) Volume 1, Issue 5, May 2014. ISSN 2348-485.

[18] M. Oliveira , B. Bowen ,R. McKenna, Yu-Sung Chang "Fast digital image inpainting" in proceeding VIIP 2001, pp. 261-266, 2001.

[19] Seema Kumari Singh, Prof J.V Shinde "Optimum Patch Selection Using GA in Exemplar Based Image Inpainting" (IJCSIT) International Journal of Computer Science and Information Technologies, Vol. 6 (2), 2015, 990-997. 